\title{
New Arythioindoles, Potent Inhibitors of Tubulin Polymerization. 2. Structure Activity Relationships and Molecular Modeling Studies
}

Gabriella De Martino, Michael C. Edler, Giuseppe La Regina, Antonio Coluccia, Maria Chiara Barbera, Denise Barrow, Robert I. Nicholson, Gabriela Chiosis, Andrea Brancale, Ernest Hamel, Marino Artico, and Romano Silvestri

${ }^{1} \mathrm{H}$ NMR and IR spectral data of new compounds 5-12, 14, 16-20, 22, 25, 27, 28, 30-34 and 36-41.

5: ${ }^{1} \mathrm{H}$ NMR $\left(\mathrm{CDCl}_{3}\right): \delta 0.96(\mathrm{t}, J=7.4 \mathrm{~Hz}, 3 \mathrm{H}), 1.68-1.74(\mathrm{~m}, 2 \mathrm{H}), 4.31(\mathrm{t}, J=6.6 \mathrm{~Hz}, 2 \mathrm{H}), 7.08-$ $7.10(\mathrm{~m}, 1 \mathrm{H}), 7.15-7.17(\mathrm{~m}, 5 \mathrm{H}), 7.36(\mathrm{t}, J=7.6 \mathrm{~Hz}, 1 \mathrm{H}), 7.46(\mathrm{~d}, J=8.3 \mathrm{~Hz}, 1 \mathrm{H}), 7.61(\mathrm{~d}, J=8.3$ $\mathrm{Hz}, 1 \mathrm{H}), 9.33 \mathrm{ppm}\left(\right.$ broad s, $1 \mathrm{H}$, disappeared on treatment with $\left.\mathrm{D}_{2} \mathrm{O}\right)$. IR: $v 1687,3288 \mathrm{~cm}^{-1}$.

6: ${ }^{1} \mathrm{H}$ NMR $\left(\mathrm{CDCl}_{3}\right): \delta 1.28(\mathrm{~d}, J=6.2 \mathrm{~Hz}, 6 \mathrm{H}), 5.22-5.28(\mathrm{~m}, 1 \mathrm{H}), 7.06-7.10(\mathrm{~m}, 1 \mathrm{H}), 7.13-7.17$ $(\mathrm{m}, 5 \mathrm{H}), 7.36(\mathrm{t}, J=7.7 \mathrm{~Hz}, 1 \mathrm{H}), 7.45(\mathrm{~d}, J=8.2 \mathrm{~Hz}, 1 \mathrm{H}), 7.64(\mathrm{~d}, J=8.2 \mathrm{~Hz}, 1 \mathrm{H}), 9.28 \mathrm{ppm}$ (broad s, $1 \mathrm{H}$, disappeared on treatment with $\mathrm{D}_{2} \mathrm{O}$ ). IR: $v 1675,3296 \mathrm{~cm}^{-1}$.

7: ${ }^{1} \mathrm{H}$ NMR $\left(\mathrm{CDCl}_{3}\right): \delta 0.90(\mathrm{t}, J=7.4 \mathrm{~Hz}, 3 \mathrm{H}), 1.37-1.42(\mathrm{~m}, 2 \mathrm{H}), 1.62-1.69(\mathrm{~m}, 2 \mathrm{H}), 4.34(\mathrm{t}, J=$ $6.5 \mathrm{~Hz}, 2 \mathrm{H}), 7.06-7.10(\mathrm{~m}, 1 \mathrm{H}), 7.13-7.19(\mathrm{~m}, 5 \mathrm{H}), 7.36(\mathrm{t}, J=7.5 \mathrm{~Hz}, 1 \mathrm{H}), 7.46(\mathrm{~d}, J=8.0 \mathrm{~Hz}$, $1 \mathrm{H}), 7.62(\mathrm{~d}, J=8.2 \mathrm{~Hz}, 1 \mathrm{H}), 9.27 \mathrm{ppm}\left(\right.$ broad s, $1 \mathrm{H}$, disappeared on treatment with $\left.\mathrm{D}_{2} \mathrm{O}\right) . \mathrm{IR}: v$ $1687,3289 \mathrm{~cm}^{-1}$.

8: ${ }^{1} \mathrm{H}$ NMR $\left(\mathrm{CDCl}_{3}\right): \delta 0.90(\mathrm{t}, J=7.4 \mathrm{~Hz}, 3 \mathrm{H}), 1.25(\mathrm{~d}, J=6.2,3 \mathrm{H}), 1.55-1.67(\mathrm{~m}, 2 \mathrm{H}), 5.08-5.13$ $(\mathrm{m}, 1 \mathrm{H}), 7.05-7.092(\mathrm{~m}, 1 \mathrm{H}), 7.13-7.17(\mathrm{~m}, 5 \mathrm{H}), 7.36(\mathrm{t}, J=8.2 \mathrm{~Hz}, 1 \mathrm{H}), 7.45(\mathrm{~d}, J=8.2 \mathrm{~Hz}, 1 \mathrm{H})$, $7.63(\mathrm{~d}, J=7.9 \mathrm{~Hz}, 1 \mathrm{H}), 9.24 \mathrm{ppm}\left(\right.$ broad s, $1 \mathrm{H}$, disappeared on treatment with $\left.\mathrm{D}_{2} \mathrm{O}\right) . \mathrm{IR}: v 1682$, $3292 \mathrm{~cm}^{-1}$.

9: ${ }^{1} \mathrm{H}$ NMR $\left(\mathrm{CDCl}_{3}\right): \delta 1.52(\mathrm{~s}, 9 \mathrm{H}), 7.05-7.09(\mathrm{~m}, 1 \mathrm{H}), 7.12-7.19(\mathrm{~m}, 5 \mathrm{H}), 7.34(\mathrm{t}, J=7.6 \mathrm{~Hz}, 1 \mathrm{H})$, $7.44(\mathrm{~d}, J=8.3 \mathrm{~Hz}, 1 \mathrm{H}), 7.62(\mathrm{~d}, J=8.3 \mathrm{~Hz}, 1 \mathrm{H}), 9.24 \mathrm{ppm}($ broad s, $1 \mathrm{H}$, disappeared on treatment with $\left.\mathrm{D}_{2} \mathrm{O}\right)$. IR: $v 1692,3307 \mathrm{~cm}^{-1}$.

10: ${ }^{1} \mathrm{H}$ NMR (DMSO- $\left.d_{6}\right)$ : $\delta 0.81(\mathrm{~d}, J=6.7 \mathrm{~Hz}, 6 \mathrm{H}), 1.47(\mathrm{q}, J=6.7 \mathrm{~Hz}, 2 \mathrm{H}), 1.62-1.69(\mathrm{~m}, 1 \mathrm{H})$, $4.27(\mathrm{t}, J=6.6 \mathrm{~Hz}, 2 \mathrm{H}), 7.00-7.03(\mathrm{~m}, 2 \mathrm{H}), 7.06-7.11(\mathrm{~m}, 2 \mathrm{H}), 7.18(\mathrm{t}, J=7.6 \mathrm{~Hz}, 2 \mathrm{H}), 7.31(\mathrm{t}, J=$ $7.6 \mathrm{~Hz}, 1 \mathrm{H}), 7.44(\mathrm{~d}, J=8.0 \mathrm{~Hz}, 1 \mathrm{H}), 7.53(\mathrm{~d}, J=8.3 \mathrm{~Hz}, 1 \mathrm{H}), 12.37 \mathrm{ppm}($ broad s, $1 \mathrm{H}$, disappeared on treatment with $\left.\mathrm{D}_{2} \mathrm{O}\right)$. IR: $v 1683,3297 \mathrm{~cm}^{-1}$.

11: ${ }^{1} \mathrm{H}$ NMR $\left(\mathrm{CDCl}_{3}\right)$ : $\delta 5.39(\mathrm{~s}, 2 \mathrm{H}), 7.08-7.17(\mathrm{~m}, 5 \mathrm{H}), 7.32-7.34(\mathrm{~m}, 5 \mathrm{H}), 7.36-7.39(\mathrm{~m}, 2 \mathrm{H})$, $7.42(\mathrm{~d}, J=8.3 \mathrm{~Hz}, 1 \mathrm{H}), 7.63(\mathrm{~d}, J=8.3 \mathrm{~Hz}, 1 \mathrm{H}), 9.31 \mathrm{ppm}$ (broad s, 1H, disappeared on treatment with $\left.\mathrm{D}_{2} \mathrm{O}\right)$. IR: $v 1681,3305 \mathrm{~cm}^{-1}$.

12: ${ }^{1} \mathrm{H}$ NMR $\left(\mathrm{CDCl}_{3}\right): \delta 1.26(\mathrm{t}, J=7.2 \mathrm{~Hz}, 3 \mathrm{H}), 3.94(\mathrm{~s}, 3 \mathrm{H}), 4.37(\mathrm{q}, J=7.2 \mathrm{~Hz}, 2 \mathrm{H}), 6.64-6.70$ $(\mathrm{m}, 2 \mathrm{H}), 6.85(\mathrm{~d}, J=8.0 \mathrm{~Hz}, 1 \mathrm{H}), 7.06(\mathrm{~m}, 1 \mathrm{H}), 7.18(\mathrm{t}, J=7.1 \mathrm{~Hz}, 1 \mathrm{H}), 7.36(\mathrm{t}, J=7.2 \mathrm{~Hz}, 1 \mathrm{H})$, $7.46(\mathrm{~d}, J=8.2 \mathrm{~Hz}, 1 \mathrm{H}), 7.64(\mathrm{~d}, J=8.2 \mathrm{~Hz}, 1 \mathrm{H}), 9.35 \mathrm{ppm}$ (broad s, 1H, disappeared on treatment with $\left.\mathrm{D}_{2} \mathrm{O}\right)$. IR: $v 1685,3311 \mathrm{~cm}^{-1}$.

14: ${ }^{1} \mathrm{H}$ NMR $\left(\mathrm{CDCl}_{3}\right): \delta 1.38(\mathrm{t}, J=7.1 \mathrm{~Hz}, 3 \mathrm{H}), 3.75(\mathrm{~s}, 3 \mathrm{H}), 4.43(\mathrm{q}, J=7.1 \mathrm{~Hz}, 2 \mathrm{H}), 6.76(\mathrm{~d}, J=$ $8.8 \mathrm{~Hz}, 2 \mathrm{H}), 7.11(\mathrm{t}, J=7.6 \mathrm{~Hz}, 1 \mathrm{H}), 7.23-7.26(\mathrm{~m}, 2 \mathrm{H}), 7.33(\mathrm{t}, J=7.6 \mathrm{~Hz}, 1 \mathrm{H}), 7.41(\mathrm{~d}, J=7.6$ $\mathrm{Hz}, 1 \mathrm{H}), 7.55(\mathrm{~d}, 7.6 \mathrm{~Hz}, 1 \mathrm{H}), 9.27 \mathrm{ppm}$ (broad s, $1 \mathrm{H}$, disappeared on treatment with $\left.\mathrm{D}_{2} \mathrm{O}\right)$. IR: $v$ 1682, $3299 \mathrm{~cm}^{-1}$. Anal. Calcd. $\left(\mathrm{C}_{18} \mathrm{H}_{17} \mathrm{NO}_{3} \mathrm{~S}(327.40)\right) \mathrm{C}, \mathrm{H}, \mathrm{N}, \mathrm{S}$.

16: ${ }^{1} \mathrm{H}$ NMR $\left(\mathrm{CDCl}_{3}\right): \delta 1.37(\mathrm{t}, J=7.1 \mathrm{~Hz}, 3 \mathrm{H}), 3.67(\mathrm{~s}, 9 \mathrm{H}), 4.30(\mathrm{q}, J=7.1 \mathrm{~Hz}, 2 \mathrm{H}), 6.47(\mathrm{~s}, 2 \mathrm{H})$, $7.14(\mathrm{t}, J=8.4 \mathrm{~Hz}, 1 \mathrm{H}), 7.34(\mathrm{t}, J=6.6 \mathrm{~Hz}, 1 \mathrm{H}), 7.44(\mathrm{~d}, J=6.2 \mathrm{~Hz}, 1 \mathrm{H}), 7.57(\mathrm{~d}, J=7.3 \mathrm{~Hz}, 1 \mathrm{H})$, $9.25 \mathrm{ppm}$ (broad s, $1 \mathrm{H}$, disappeared on treatment with $\left.\mathrm{D}_{2} \mathrm{O}\right)$. IR: $v 1683,3313 \mathrm{~cm}^{-1}$.

17: ${ }^{1} \mathrm{H}$ NMR (DMSO- $\left.d_{6}\right)$ : $\delta 3.83(\mathrm{~s}, 3 \mathrm{H}), 3.86(\mathrm{~s}, 3 \mathrm{H}), 6.42(\mathrm{dd}, J=1.3$ and $7.8 \mathrm{~Hz}, 1 \mathrm{H}), 6.69(\mathrm{t}, J=$ $7.3 \mathrm{~Hz}, 1 \mathrm{H}), 6.99(\mathrm{~d}, J=7.9 \mathrm{~Hz}, 1 \mathrm{H}), 7.07(\mathrm{t}, J=7.9,1 \mathrm{H}), 7.30-7.33(\mathrm{~m}, 2 \mathrm{H}), 7.54(\mathrm{~d}, J=8.6 \mathrm{~Hz}$, $1 \mathrm{H}), 12.63 \mathrm{ppm}$ (broad s, $1 \mathrm{H}$, disappeared on treatment with $\left.\mathrm{D}_{2} \mathrm{O}\right)$. IR: $v 1684,3288 \mathrm{~cm}^{-1}$. 
18: ${ }^{1} \mathrm{H}$ NMR (DMSO- $\left.d_{6}\right): \delta 3.49(\mathrm{~s}, 3 \mathrm{H}), 3.76(\mathrm{~s}, 3 \mathrm{H}), 7.08(\mathrm{~d}, J=8.3 \mathrm{~Hz}, 1 \mathrm{H}), 7.17(\mathrm{t}, J=7.6 \mathrm{~Hz}$, $1 \mathrm{H}), 7.40(\mathrm{dd}, J=2.0$ and $8.8 \mathrm{~Hz}, 1 \mathrm{H}), 7.54-7.62(\mathrm{~m}, 2 \mathrm{H}), 8.02(\mathrm{dd}, J=1.6$ and $7.8 \mathrm{~Hz}, 1 \mathrm{H}), 8.25$ $(\mathrm{d}, J=1.6 \mathrm{~Hz}, 1 \mathrm{H}), 13.33 \mathrm{ppm}$ (broad s, $1 \mathrm{H}$, disappeared on treatment with $\mathrm{D}_{2} \mathrm{O}$ ). IR: $v 1737,1709$, $3284 \mathrm{~cm}^{-1}$.

19: ${ }^{1} \mathrm{H}$ NMR (DMSO- $\left.d_{6}\right)$ : $\delta 3.64(\mathrm{~s}, 3 \mathrm{H}), 3.85(\mathrm{~s}, 3 \mathrm{H}), 6.57-6.61(\mathrm{~m}, 2 \mathrm{H}), 6.62(\mathrm{~d}, J=1.9 \mathrm{~Hz}, 1 \mathrm{H})$, $7.12(\mathrm{t}, J=8.0 \mathrm{~Hz}, 1 \mathrm{H}), 7.31-7.35(\mathrm{~m}, 2 \mathrm{H}), 7.53(\mathrm{~d}, J=8.7 \mathrm{~Hz}, 1 \mathrm{H}), 12.72 \mathrm{ppm}$ (broad s, $1 \mathrm{H}$, disappeared on treatment with $\left.\mathrm{D}_{2} \mathrm{O}\right)$. IR: $v 1683,3295 \mathrm{~cm}^{-1}$.

20: ${ }^{1} \mathrm{H}$ NMR (DMSO- $\left.d_{6}\right)$ : $\delta 3.79(\mathrm{~s}, 3 \mathrm{H}), 3.88(\mathrm{~s}, 3 \mathrm{H}), 7.20(\mathrm{~d}, J=8.2 \mathrm{~Hz}, 1 \mathrm{H}), 7.42(\mathrm{dd}, J=2.1$ and $8.2 \mathrm{~Hz}, 1 \mathrm{H}), 7.49(\mathrm{t}, J=8.2 \mathrm{~Hz}, 1 \mathrm{H}), 7.54-7.59(\mathrm{~m}, 3 \mathrm{H}), 8.19(\mathrm{t}, J=1.9 \mathrm{~Hz}, 1 \mathrm{H}), 13.18 \mathrm{ppm}$ (broad s, 1H, disappeared on treatment with $\mathrm{D}_{2} \mathrm{O}$ ). IR: $v 1696,3272 \mathrm{~cm}^{-1}$.

22: ${ }^{1} \mathrm{H}$ NMR (DMSO- $\left.d_{6}\right)$ : $\delta 3.79(\mathrm{~s}, 3 \mathrm{H}), 3.89(\mathrm{~s}, 3 \mathrm{H}), 7.09(\mathrm{~d}, J=8.9 \mathrm{~Hz}, 2 \mathrm{H}), 7.41(\mathrm{dd}, J=1.9$ and $8.9 \mathrm{~Hz}, 1 \mathrm{H}), 7.56(\mathrm{~d}, J=8.9 \mathrm{~Hz}, 1 \mathrm{H}), 7.94(\mathrm{~d}, J=8.9 \mathrm{~Hz}, 2 \mathrm{H}), 8.22(\mathrm{~d}, J=1.9 \mathrm{~Hz}, 1 \mathrm{H}), 13.34 \mathrm{ppm}$ (broad s, 1H, disappeared on treatment with $\mathrm{D}_{2} \mathrm{O}$ ). IR: $v 1682,3311 \mathrm{~cm}^{-1}$.

25: ${ }^{1} \mathrm{H}$ NMR (DMSO- $\left.d_{6}\right): \delta 3.61(\mathrm{~s}, 6 \mathrm{H}), 3.86(\mathrm{~s}, 3 \mathrm{H}), 6.14(\mathrm{~d}, J=1.9 \mathrm{~Hz}, 2 \mathrm{H}), 6.26(\mathrm{t}, J=1.9 \mathrm{~Hz}$, $1 \mathrm{H}), 7.33(\mathrm{dd}, J=1.9$ and $8.8 \mathrm{~Hz}, 1 \mathrm{H}), 7.37(\mathrm{~d}, J=1.9 \mathrm{~Hz}, 1 \mathrm{H}), 7.54(\mathrm{~d}, J=8.8 \mathrm{~J} \mathrm{~Hz}, 1 \mathrm{H}), 12.66$ ppm (broad s, $1 \mathrm{H}$, disappeared on treatment with $\mathrm{D}_{2} \mathrm{O}$ ). IR: $v 1687,3312 \mathrm{~cm}^{-1}$.

27: ${ }^{1} \mathrm{H}$ NMR (DMSO- $\left.d_{6}\right): \delta 1.27(\mathrm{t}, J=7.1 \mathrm{~Hz}, 3 \mathrm{H}), 3.59(\mathrm{~s}, 9 \mathrm{H}), 4.33(\mathrm{q}, J=7.1 \mathrm{~Hz}, 2 \mathrm{H}), 6.47$ (s, $2 \mathrm{H}), 7.31(\mathrm{~d}, J=8.7 \mathrm{~Hz}, 1 \mathrm{H}), 7.36(\mathrm{~s}, 1 \mathrm{H}), 7.52(\mathrm{~d}, J=8.7 \mathrm{~Hz}, 1 \mathrm{H}), 12.51 \mathrm{ppm}($ broad s, $1 \mathrm{H}$, disappeared on treatment with $\left.\mathrm{D}_{2} \mathrm{O}\right)$. IR: $v 1675,3300 \mathrm{~cm}^{-1}$. Anal. Calcd. $\left(\mathrm{C}_{20} \mathrm{H}_{20} \mathrm{ClNO}_{5} \mathrm{~S}(421.90)\right)$ $\mathrm{C}, \mathrm{H}, \mathrm{Cl}, \mathrm{N}, \mathrm{S}$.

28: ${ }^{1} \mathrm{H}$ NMR $\left(\mathrm{CDCl}_{3}\right): \delta 3.89(\mathrm{~s}, 3 \mathrm{H}), 3.92(\mathrm{~s}, 3 \mathrm{H}), 6.98(\mathrm{~d}, J=8.8 \mathrm{~Hz}, 1 \mathrm{H}), 7.05-7.11(\mathrm{~m}, 3 \mathrm{H})$, 7.16-7.20 (m, 2H), $7.21(\mathrm{~d}, J=2.28 \mathrm{~Hz}, 1 \mathrm{H}), 7.74(\mathrm{~d}, J=8.8 \mathrm{~Hz}, 1 \mathrm{H}), 8.97 \mathrm{ppm}$ (broad s, $1 \mathrm{H}$, disappeared on treatment with $\left.\mathrm{D}_{2} \mathrm{O}\right)$. IR: $v 1700,3329 \mathrm{~cm}^{-1}$. Anal. Calcd. $\left(\mathrm{C}_{17} \mathrm{H}_{15} \mathrm{NO}_{3} \mathrm{~S}(313.38)\right) \mathrm{C}$, $\mathrm{H}, \mathrm{N}, \mathrm{S}$.

30: ${ }^{1} \mathrm{H}$ NMR (DMSO- $\left.d_{6}\right)$ : $\delta 3.83(\mathrm{~s}, 6 \mathrm{H}), 7.05(\mathrm{dd}, J=2.4$ and $9.1 \mathrm{~Hz}, 1 \mathrm{H}), 7.45(\mathrm{~d}, \mathrm{~J}=9.1 \mathrm{~Hz}, 1 \mathrm{H})$, 7.54-7.63 (m, 3H), $7.67(\mathrm{~d}, J=2.4 \mathrm{~Hz}, 1 \mathrm{H}), 7.99(\mathrm{~d}, J=9.1 \mathrm{~Hz}, 2 \mathrm{H}), 13,22 \mathrm{ppm}$ (broad s, $1 \mathrm{H}$, disappeared on treatment with $\left.\mathrm{D}_{2} \mathrm{O}\right)$. IR: $v 1733,3246 \mathrm{~cm}^{-1}$.

31: ${ }^{1} \mathrm{H}$ NMR (DMSO- $\left.d_{6}\right): \delta 3.82(\mathrm{~s}, 3 \mathrm{H}), 3.94(\mathrm{~s}, 3 \mathrm{H}), 6.70(\mathrm{~d}, J=8.1 \mathrm{~Hz}, 1 \mathrm{H}), 6.98-7.00(\mathrm{~m}, 2 \mathrm{H})$, $7.10(\mathrm{t}, J=7.9 \mathrm{~Hz}, 1 \mathrm{H}), 7.20-7.24(\mathrm{~m}, 3 \mathrm{H}), 7.47(\mathrm{~d}, J=8.0 \mathrm{~Hz}, 1 \mathrm{H}), 11.81 \mathrm{ppm}($ broad s, $1 \mathrm{H}$, disappeared on treatment with $\left.\mathrm{D}_{2} \mathrm{O}\right)$. IR: $v 1702,3325 \mathrm{~cm}^{-1}$.

32: ${ }^{1} \mathrm{H}$ NMR $\left(\mathrm{CDCl}_{3}\right): \delta 1.22(\mathrm{t}, J=7.1 \mathrm{~Hz}, 3 \mathrm{H}), 3.74(\mathrm{~s}, 3 \mathrm{H}), 3.95(\mathrm{~s}, 3 \mathrm{H}), 4.34(\mathrm{q}, J=7.1 \mathrm{~Hz}, 2 \mathrm{H})$, 6.64-6.72 (m, 2H), $6.86(\mathrm{~d}, J=7.9 \mathrm{~Hz}, 1 \mathrm{H}), 7.00-7.08(\mathrm{~m}, 3 \mathrm{H}), 7.35(\mathrm{dd}, J=2.3$ and $7.5 \mathrm{~Hz}, 1 \mathrm{H})$, $9.39 \mathrm{ppm}$ (broad s, $1 \mathrm{H}$, disappeared on treatment with $\mathrm{D}_{2} \mathrm{O}$ ). IR: $v 1680,3275 \mathrm{~cm}^{-1}$. Anal. Calcd. $\left(\mathrm{C}_{19} \mathrm{H}_{19} \mathrm{NO}_{4} \mathrm{~S}(357.43)\right) \mathrm{C}, \mathrm{H}, \mathrm{N}, \mathrm{S}$.

33: ${ }^{1} \mathrm{H}$ NMR $\left(\mathrm{CDCl}_{3}\right)$ : $\delta 1.30(\mathrm{t}, J=7.1 \mathrm{~Hz}, 3 \mathrm{H}), 3.69(\mathrm{~s}, 3 \mathrm{H}), 3.75(\mathrm{~s}, 3 \mathrm{H}), 4.37(\mathrm{q}, J=7.1 \mathrm{~Hz}, 2 \mathrm{H})$, $6.63(\mathrm{dd}, J=2.4$ and $7.9 \mathrm{~Hz}, 1 \mathrm{H}), 6.71(\mathrm{t}, J=2.1 \mathrm{~Hz}, 1 \mathrm{H}), 6.74(\mathrm{~d}, J=7.9 \mathrm{~Hz}, 1 \mathrm{H}), 7.00(\mathrm{~s}, 1 \mathrm{H})$, $7.03(\mathrm{~d}, J=2.4 \mathrm{~Hz}, 1 \mathrm{H}), 7.09(\mathrm{t}, J=7.9 \mathrm{~Hz}, 1 \mathrm{H}), 7.33(\mathrm{dd}, J=1.3$ and $8.2 \mathrm{~Hz}, 1 \mathrm{H}), 9.31 \mathrm{ppm}$ (broad s, 1H, disappeared on treatment with $\left.\mathrm{D}_{2} \mathrm{O}\right)$. IR: $v 1678,3308 \mathrm{~cm}^{-1}$.

34: ${ }^{1} \mathrm{H}$ NMR $\left(\mathrm{CDCl}_{3}\right): \delta 1.36(\mathrm{t}, J=7.1 \mathrm{~Hz}, 3 \mathrm{H}), 3.73(\mathrm{~s}, 3 \mathrm{H}), 3.75(\mathrm{~s}, 3 \mathrm{H}), 4.40(\mathrm{q}, J=7.1 \mathrm{~Hz}, 2 \mathrm{H})$, $6.76(\mathrm{~d}, J=8.8 \mathrm{~Hz}, 2 \mathrm{H}), 7.23-7.31(\mathrm{~m}, 5 \mathrm{H}), 9.09 \mathrm{ppm}$ (broad s, $1 \mathrm{H}$, disappeared on treatment with $\left.\mathrm{D}_{2} \mathrm{O}\right)$. IR: $v 1676,3311 \mathrm{~cm}^{-1}$.

36: ${ }^{1} \mathrm{H}$ NMR (DMSO- $d_{6}$ ): $\delta 1.34(\mathrm{t}, J=7.1 \mathrm{~Hz}, 3 \mathrm{H}), 3.69(\mathrm{~s}, 6 \mathrm{H}), 3.76(\mathrm{~s}, 3 \mathrm{H}), 3.78(\mathrm{~s}, 3 \mathrm{H}), 4.40$ $(\mathrm{q}, J=7.1 \mathrm{~Hz}, 2 \mathrm{H}), 6.48(\mathrm{~s}, 2 \mathrm{H}), 6.95(\mathrm{~d}, J=2.4 \mathrm{~Hz}, 1 \mathrm{H}), 7.01(\mathrm{dd}, J=2.4$ and $8.9 \mathrm{~Hz}, 1 \mathrm{H}), 7.33$ $(\mathrm{d}, J=8.9 \mathrm{~Hz}, 1 \mathrm{H}), 9.15 \mathrm{ppm}\left(\right.$ broad s, $1 \mathrm{H}$, disappeared on treatment with $\left.\mathrm{D}_{2} \mathrm{O}\right)$. IR: $v 1672,3294$ $\mathrm{cm}^{-1}$. Anal. Calcd. $\left(\mathrm{C}_{21} \mathrm{H}_{23} \mathrm{NO}_{6} \mathrm{~S}(417.48)\right) \mathrm{C}, \mathrm{H}, \mathrm{N}, \mathrm{S}$.

37: ${ }^{1} \mathrm{H}$ NMR $\left(\mathrm{CDCl}_{3}\right): \delta 3.74(\mathrm{~s}, 6 \mathrm{H}), 3.82(\mathrm{~s}, 3 \mathrm{H}), 4.01(\mathrm{~s}, 3 \mathrm{H}), 6.61(\mathrm{~s}, 2 \mathrm{H}), 7.49(\mathrm{~d}, J=9.0 \mathrm{~Hz}$, $1 \mathrm{H}), 8.21(\mathrm{dd}, J=2.1$ and $9.0 \mathrm{~Hz}, 1 \mathrm{H}), 8.40(\mathrm{~d}, J=2.1 \mathrm{~Hz}, 1 \mathrm{H}), 9.58 \mathrm{ppm}$ (broad s, $1 \mathrm{H}$, disappeared on treatment with $\left.\mathrm{D}_{2} \mathrm{O}\right)$. IR: $v 1678,3287 \mathrm{~cm}^{-1}$. Anal. Calcd. $\left(\mathrm{C}_{19} \mathrm{H}_{18} \mathrm{~N}_{2} \mathrm{O}_{7} \mathrm{~S}(418.43)\right) \mathrm{C}$, $\mathrm{H}, \mathrm{N}, \mathrm{S}$. 
38: ${ }^{1} \mathrm{H}$ NMR (DMSO- $\left.d_{6}\right)$ : $\delta 1.17(\mathrm{t}, J=7.1 \mathrm{~Hz}, 3 \mathrm{H}), 3.62(\mathrm{~s}, 3 \mathrm{H}), 3.79(\mathrm{~s}, 3 \mathrm{H}), 4.23(\mathrm{q}, J=7.1 \mathrm{~Hz}$, 2H), $6.75(\mathrm{~s}, 1 \mathrm{H}), 6.94(\mathrm{~s}, 1 \mathrm{H}), 7.04-7.10(\mathrm{~m}, 3 \mathrm{H}), 7.20(\mathrm{t}, J=7.6 \mathrm{~Hz}, 2 \mathrm{H}), 12.09 \mathrm{ppm}$ (broad s, 1H, disappeared on treatment with $\left.\mathrm{D}_{2} \mathrm{O}\right)$. IR: $v 1675,3306 \mathrm{~cm}^{-1}$.

39: ${ }^{1} \mathrm{H}$ NMR (DMSO- $\left.d_{6}\right)$ : $\delta 1.22(\mathrm{t}, J=7.1 \mathrm{~Hz}, 3 \mathrm{H}), 3.81(\mathrm{~s}, 3 \mathrm{H}), 3.84(\mathrm{~s}, 3 \mathrm{H}), 4.26(\mathrm{q}, J=7.1 \mathrm{~Hz}$, 2H), $6.96(\mathrm{~s}, 1 \mathrm{H}), 7.53-7.66(\mathrm{~m}, 4 \mathrm{H}), 7.94(\mathrm{~d}, J=7.2 \mathrm{~Hz}, 2 \mathrm{H}), 12.38 \mathrm{ppm}$ (broad s, 1H, disappeared on treatment with $\left.\mathrm{D}_{2} \mathrm{O}\right)$. IR: $v 1723,3360 \mathrm{~cm}^{-1}$.

40: ${ }^{1} \mathrm{H}$ NMR $\left(\mathrm{CDCl}_{3}\right)$ : $\delta 3.69$ (s, 6H), 3.78 (s, 6H), 3.93 (s, 6H), 6.49 (s, 2H), $6.83(\mathrm{~s}, 2 \mathrm{H}), 9.03$ ppm (broad s, 1H, disappeared on treatment with $\mathrm{D}_{2} \mathrm{O}$ ). IR: $v 1681,3283 \mathrm{~cm}^{-1}$.

41: ${ }^{1} \mathrm{H}$ NMR (DMSO- $\left.d_{6}\right): \delta 1.16(\mathrm{t}, J=7.0 \mathrm{~Hz}, 3 \mathrm{H}), 3.34(\mathrm{~s}, 3 \mathrm{H}), 3.69(\mathrm{~s}, 3 \mathrm{H}), 6.69(\mathrm{~s}, 3 \mathrm{H}), 3.79$ (s, $6 \mathrm{H}), 4.23(\mathrm{q}, J=7.0 \mathrm{~Hz}, 2 \mathrm{H}), 6.12(\mathrm{~s}, 2 \mathrm{H}), 6.77(\mathrm{~s}, 1 \mathrm{H}), 6.95(\mathrm{~s}, 1 \mathrm{H}), 12.20 \mathrm{ppm}$ (broad s, 1H, disappeared on treatment with $\left.\mathrm{D}_{2} \mathrm{O}\right)$. IR: $v 1665,3296 \mathrm{~cm}^{-1}$.

$\boldsymbol{N}$-(2-Methoxyphenylthio)succinimide: ${ }^{1} \mathrm{H}$ NMR $\left(\mathrm{CDCl}_{3}\right): \delta 3.45$ (s, 3H), $4.03(\mathrm{~s}, 4 \mathrm{H}), 7.10 \mathrm{ppm}$ (m, 4H) IR (Nujol): $v 1377,1590 \mathrm{~cm}^{-1}$.

$N$-(4-Methoxyphenylthio)succinimide: ${ }^{1} \mathrm{H}$ NMR $\left(\mathrm{CDCl}_{3}\right): \delta 2.75(\mathrm{~s}, 4 \mathrm{H}), 3.79(\mathrm{~s}, 3 \mathrm{H}), 6.84(\mathrm{~d}, J=$ $8.5,2 \mathrm{H}), 7.74 \mathrm{ppm}(\mathrm{d}, J=8.5 \mathrm{~Hz}, 2 \mathrm{H})$. IR $(\mathrm{KBr}): v 1592,1721,2926 \mathrm{~cm}^{-1}$. 
Appendix. Elemental Analyses of New Compounds 5-12, 14, 16-20, 22, 25, 27, 28, 30-34 and 36-41.

\begin{tabular}{llll}
\hline \multirow{2}{*}{ Compd } & Calculated & Found \\
\cline { 2 - 2 } & $\mathrm{C}, \mathrm{H}, \mathrm{N}, \mathrm{Cl}, \mathrm{S}$ & $\mathrm{C}, \mathrm{H}, \mathrm{N}, \mathrm{Cl}, \mathrm{S}$ \\
\hline $\mathbf{5}$ & $69.43,5.50,4.50,-, 10.30$ & & $69.37,5.47,4.47,-, 10.21$ \\
$\mathbf{6}$ & $69.43,5.50,4.50,-, 10.30$ & & $69.20,5.46,4.38,-, 10.22$ \\
$\mathbf{7}$ & $70.12,5.88,4.30,-, 9.85$ & & $70.04,5.80,4.21,-, 9.80$ \\
$\mathbf{8}$ & $70.12,5.88,4.30,-, 9.85$ & $69.96,5.77,4.22,-, 9.72$ \\
$\mathbf{9}$ & $70.12,5.88,4.30,-, 9.85$ & $69.83,5.81,4.06,-, 9.57$ \\
$\mathbf{1 0}$ & $70.77,6.24,4.13,-, 9.45$ & & $70.53,6.19,3.94,-, 9.22$ \\
$\mathbf{1 1}$ & $73.51,4.77,3.90,-, 8.92$ & $73.34,4.72,3.82,-, 8.88$ \\
$\mathbf{1 2}$ & $66.03,5.23,4.28,-, 9.79$ & $65.81,5.20,4.21,-, 9.57$ \\
$\mathbf{1 4}$ & $66.03,5.23,4.28,-, 9.79$ & $65.91,5.21,4.16,-, 9.66$ \\
$\mathbf{1 6}$ & $62.00,5.46,3.62,-, 8.28$ & $61.87,5.38,3.44,-, 8.03$ \\
$\mathbf{1 7}$ & $58.70,4.06,4.03,10.19,9.22$ & $58.55,3.98,3.88,10.03,9.18$ \\
$\mathbf{1 8}$ & $53.76,3.72,3.69,9.33,8.44$ & $53.70,3.72,3.61,9.18,8.29$ \\
$\mathbf{1 9}$ & $58.70,4.06,4.03,10.19,9.22$ & $58.45,3.92,3.87,10.22,9.07$ \\
$\mathbf{2 0}$ & $53.76,3.72,3.69,9.33,8.44$ & $53.48,3.55,3.44,9.17,8.27$ \\
$\mathbf{2 2}$ & $53.76,3.72,3.69,9.33,8.44$ & $53.55,3.62,3.44,9.22,8.31$ \\
$\mathbf{2 5}$ & $57.22,4.27,3.71,9.38,8.49$ & $57.12,4.24,3.70,9.32,8.44$ \\
$\mathbf{2 7}$ & $56.94,4.78,3.32,8.40,7.60$ & $57.02,4.80,3.13,8.26,7.49$ \\
$\mathbf{2 8}$ & $65.16,4.82,4.47,-, 10.23$ & $64.98,4.77,4.38,-, 10.06$ \\
$\mathbf{3 0}$ & $59.12,4.38,4.06,-, 9.28$ & $58.96,4.33,3.91,-, 9.12$ \\
$\mathbf{3 1}$ & $65.16,4.82,4.47,-, 10.23$ & $65.08,4.80,4.41,-, 9.95$ \\
$\mathbf{3 2}$ & $63.85,5.36,3.92,-, 8.97$ & $63.72,5.28,3.77,-, 8.65$ \\
$\mathbf{3 3}$ & $63.85,5.36,3.92,-, 8.97$ & $63.88,5.35,3.87,-, 8.88$ \\
$\mathbf{3 4}$ & $63.85,5.36,3.92,-, 8.97$ & $63.80,5.33,3.90,-, 8.91$ \\
$\mathbf{3 6}$ & $60.42,5.55,3.36,-, 7.68$ & $60.38,5.53,3.20,-, 7.58$ \\
$\mathbf{3 7}$ & $54.54,4.34,6.70,-, 7.66$ & $54.47,4.30,6.62,-, 7.60$ \\
$\mathbf{3 8}$ & $63.85,5.36,3.92,-, 8.97$ & $63.68,5.20,3.78,-, 8.91$ \\
$\mathbf{3 9}$ & $58.60,4.92,3.60,-, 8.23$ & $58.53,4.89,3.51,-, 8.21$ \\
$\mathbf{4 0}$ & $58.19,5.35,3.23,-, 7.40$ & $57.96,5.27,3.28,-, 7.28$ \\
$\mathbf{4 1}$ & $59.05,5.63,3.13,-, 7.17$ & $58.88,5.58,3.01,-, 6.98$ \\
\hline & &
\end{tabular}

